\title{
Use of intraoperative Doppler ultrasound with neuronavigation to guide arteriovenous malformation resection: a pediatric case series
}

\author{
James S. Walkden, BMedSci, MBChB, FRCS(SN), Zsolt Zador, MD, PhD, MRCS(Eng), \\ Amit Herwadkar, MBBS, FRCR, and lan D. Kamaly-AsI, MD, MBChB, FRCS(SN) \\ Departments of Neurosurgery and Neuroradiology, Royal Manchester Children's Hospital, Manchester, United Kingdom
}

OBJECT Over the last 20 years, several intraoperative adjuncts, including ultrasonography, neuronavigation, and angiography, have been said to aid the intraoperative localization and resection of cerebral arteriovenous malformations (AVMs). The authors assessed the value of intraoperative Doppler ultrasonography in conjunction with neuronavigation during surgery for cerebral AVMs in the pediatric population.

METHODS The authors reviewed all cranial AVM resections performed by a single surgeon at their institution in the period from 2007 to 2013 and here describe their experience and results in a series of 20 consecutive AVM resections in 19 pediatric patients. Intraoperative Doppler ultrasonography had been used in conjunction with preoperative CT or neuronavigational MRI. Preoperative and postoperative clinical findings, patient age, and Spetzler-Martin AVM grade were identified in all patients.

RESULTS All patients, whose ages ranged from 2 to 16 years, underwent craniotomy and excision of an AVM, which was supratentorial in 18 cases and infratentorial in 2. Patients in 11 cases underwent preoperative embolization, and all other patients underwent cerebral angiography prior to surgery, except for 2 patients who were urgently surgically treated because of low Glasgow Coma Scale scores and associated hematoma. Spetzler-Martin Grades I (3 cases), II (6), III (7), and IV (4) AVMs were represented in this series. Intraoperative Doppler ultrasound provided high-quality images in all cases and demonstrated the location, size, and flow characteristics of the AVM and any associated hematoma. Delayed postoperative cerebral angiography demonstrated successful AVM resection in all cases. An assessment of clinical outcomes revealed no new long-term neurological deficits at 3 months postoperatively.

CONCLUSIONS Intraoperative Doppler ultrasonography is a reliable and useful tool for intraoperative localization and guidance for AVM resection in the pediatric population. When used in conjunction with neuronavigation equipment and modern microscopes, this technique has shown a very high complete resection rate with extremely low associated morbidity.

http://thejns.org/doi/abs/10.3171/2014.10.PEDS14249

KEY WORDS pediatric; arteriovenous malformation; intraoperative ultrasound; neuronavigation; Doppler ultrasonography; vascular disorders

A RTERIOVENOUS malformations (AVMs) are responsible for around $30 \%-50 \%$ of hemorrhagic strokes in the pediatric population..$^{11,13,17,18}$ These lesions can be extremely challenging to manage given the higher incidence of hemorrhagic presentation and accompanying morbidity and mortality as well as the long potential lifespan of the pediatric patient. ${ }^{13,16-18}$ Arteriovenous malformations in the pediatric population have generally been associated with higher morbidity and mortality than those in adults, ${ }^{11,18}$ although a recent study suggests that surgery may improve long-term outcomes. ${ }^{21}$ There is also a well-described phenomenon of AVM recurrence following negative postresection angiography in the pediatric population..$^{7,11,18}$ Nonetheless, pediatric AVMs have been treated surgically, with endovascular embolization, with radiosurgery, and increasingly with multimodality treatments with good results. ${ }^{5,16-18}$

The neurosurgical application of intraoperative ultra-

ABBREVIATIONS AVM = arteriovenous malformation; $\mathrm{DSA}=$ digital subtraction angiography; $\mathrm{GCS}=$ Glasgow Coma Scale; $\mathrm{MCA}=$ middle cerebral artery; $\mathrm{mRS}=$ modified Rankin Scale; $S M=$ Spetzler-Martin.

SUBMITTED May 21, 2014. ACCEPTED October 23, 2014.

INCLUDE WHEN CITING Published online December 19, 2014; DOI: 10.3171/2014.10.PEDS14249.

DISCLOSURE The authors report no conflict of interest concerning the materials or methods used in this study or the findings specified in this paper. 
sound to identify and access intracranial lesions began in the 1980s, and the technology is now used for a range of cranial lesions, including tumor, and in CSF surgery. ${ }^{12}$ The first descriptions of color Doppler ultrasonography use in surgery for cerebral vascular lesions appeared in the late 1980s and 1990s. Despite the limited technology of the time, it was possible to identify lesions, associated hematomas, and significant draining and feeding vessels to aid complete resection. ${ }^{2,20,25}$ Since then there has been a steady increase in the use of technology to aid resection of cerebral AVMs, including intraoperative ultrasound, neuronavigation, and angiography. Increasingly two or more of these technologies have been combined intraoperatively to take advantage of each modality's benefits. Several authors have described ultrasound with and without intravenous contrast in AVM surgery in adults to aid resection, to check the extent of resection, and to increase overall surgical confidence. ${ }^{4,6,8,10,24,26,28}$ Other authors have reported using neuronavigational software in conjunction with intraoperative ultrasound., ${ }^{1,14,22}$ Intraoperative angiography or intraoperative indocyanine green video-angiography (ICGA) has also been used in conjunction with intraoperative Doppler ultrasound to aid AVM resection. ${ }^{19,23,27}$ At our institution we have found that using microscope headsup display integration (OPMI Pentero, Carl Zeiss Ltd.) of frameless neuronavigation (Brainlab AG) linked to a high-resolution color Doppler ultrasound system (Hitachi Aloka ProSound Alpha 7) is extremely useful in guiding AVM resection.

\section{Methods}

We reviewed all cranial AVM resections performed at our institution by a single surgeon in the period from 2007 to 2013 and identified 20 resections in 19 consecutive patients. Data, including preoperative neurological status, age, presenting symptoms, Spetzler-Martin (SM) grade, AVM location, and any previous treatment, were collected. Intraoperative Doppler ultrasound was used in combination with neuronavigation software in all cases to aid localization and ensure complete AVM resection. All preoperative imaging and interventional procedures were recorded. Intraoperative ultrasound and screenshots from the neuronavigation software had been prospectively collected for the patient hospital records. Any immediate postoperative neurological deficits were recorded along with the modified Rankin Scale (mRS) score at 6 months. Postoperative digital subtraction angiography (DSA) was performed at approximately 6 months to establish the extent of AVM resection, which was reported by our senior neuroradiology author independently of the surgical team.

Intraoperative Doppler ultrasound provided high-quality images in all cases and demonstrated AVM location, size, flow characteristics and any associated hematoma or residual nidus. Neuronavigation software linked to our operating microscope with heads-up display also allowed for precise craniotomy positioning and minimal surrounding brain retraction during the resections. Ultrasound could also be used to adjust the neuronavigation accuracy intraoperatively to allow for brain shift due to AVM resection or CSF loss.

\section{Results}

A summary of all AVM resections performed at our institution is shown in Table 1, arranged in order of presentation, and each has been assigned a case number for ease of reference throughout this paper.

All patients, whose ages ranged from 2 to 16 years, ultimately underwent craniotomy and excision of an AVM, which was supratentorial in 18 cases and infratentorial in 2 . The majority of cases $(16$ [80\%]) presented with hemorrhage, which was associated with a decreased level of consciousness in 8 cases. Other presentations included incidental findings ( 2 cases) - including hereditary hemorrhagic telangiectasia in 1 patient (Case 19). Additional presentations were seizures (Cases 5 and 10) and a new AVM found at follow-up (Case 15). Spetzler-Martin Grades I (3 cases), II (6), III (7), and IV (4) AVMs were represented in this series. Preoperative DSA was performed in 18 cases, but was not possible in Cases 2 and 6 because low Glasgow Coma Scale (GCS) scores from significant hematomas associated with the AVMs prompted urgent surgical evacuation. In these cases the same intraoperative techniques were used, and the underlying AVM was confirmed with intraoperative Doppler ultrasound rather than preoperative angiography. Patients in 11 cases underwent successful embolization prior to surgery; the patient in Case 8 underwent two partial embolizations before surgery. Attempted embolizations were unsuccessful in 3 cases, and in 1 of them, Case 7, only an arterial flow aneurysm was believed to be safe for obliteration. In the remaining 6 cases it was believed, after the initial angiogram, that it was not safe to attempt any embolization procedure. The only angiographic complications encountered in this series were a small intraprocedural hemorrhage (Case 8, during the second DSA study) and transient unilateral deafness (Case 9).

Arteriovenous malformations in the pediatric population have generally been associated with higher morbidity and mortality than those in adults; ${ }^{10,17}$ thus, we believed it was critical to consider functional outcome in our series. As demonstrated in Table 2, 50\% of cases had a demonstrable neurological deficit (8) or seizures (2) prior to surgery. Most of the neurological findings were contralateral hemiparesis (7), although 1 case of disabling quadrantanopia was noted as well.

As can be seen in Table 2, after surgery there was 1 case (Case 6) in which the neurological deficit improved, which was probably related to the urgent evacuation of an associated AVM hematoma. Of the 8 cases with a demonstrable preoperative neurological deficit, 6 had improvement at the 6-month review; 2 cases (Cases 1 and 14) had unchanged neurology at 6 months. Immediately postoperatively 2 cases (17 and 19) had new neurological deficits, contralateral hemiparesis in both cases. Both of these patients were treated with 5 days of corticosteroids, and at 6 months postoperatively no neurological deficits were noted. Of the 2 patients presenting with seizures, 1 (Case 5) was able to successfully stop anticonvulsant medication 6 months after surgery; the other patient (Case 10) has seizure control with medication. Overall, at the 6-month review, all patients had a high level of functionality, as measured using the mRS, with most (95\% of cases) hav- 
TABLE 1. Summary of patient and lesion characteristics in 20 cases of AVM resected with the aid of ultrasonography

\begin{tabular}{|c|c|c|c|c|c|c|c|}
\hline $\begin{array}{l}\text { Case } \\
\text { No. }\end{array}$ & $\begin{array}{c}\text { Age at } \\
\text { Presentation (yrs) }\end{array}$ & $\begin{array}{c}\text { Yr of } \\
\text { Presentation }\end{array}$ & Presentation & Location & $\begin{array}{l}\text { Preop SM } \\
\text { Grade }\end{array}$ & $\begin{array}{l}\text { Preop DSA } \\
\text { Performed }\end{array}$ & $\begin{array}{c}\text { Preop Embolization } \\
\text { Performed }\end{array}$ \\
\hline 1 & 15 & 2007 & Hemorrhage/low GCS score & Posterior fossa & IV & Yes & Yes \\
\hline 2 & 15 & 2008 & Hemorrhage/GCS & Lt frontal & II & No & No \\
\hline 3 & 13 & 2008 & Hemorrhage/GCS & Rt parietal & III & Yes & Yes \\
\hline 4 & 14 & 2008 & Hemorrhage & Lt frontal & II & Yes & Unable \\
\hline 5 & 13 & 2008 & Seizure & Lt frontal & II & Yes & No \\
\hline 6 & 8 & 2009 & Hemorrhage/GCS & Rt frontal & III & No & No \\
\hline 7 & 11 & 2009 & Hemorrhage & Rt parietal & II & Yes & Flow aneurysm only \\
\hline 8 & 7 & 2009 & Hemorrhage & Lt frontal & II & Yes & 2 partial embolizations \\
\hline 9 & 12 & 2011 & Hemorrhage & Rt cerebellar & III & Yes & Yes \\
\hline 10 & 11 & 2011 & Hemorrhage/seizure & Lt parietal & III & Yes & Yes \\
\hline 11 & 6 & 2011 & Hemorrhage/GCS & Lt parietal & IV & Yes & Yes \\
\hline 12 & 9 & 2011 & Hemorrhage/GCS & Rt parietal & IV & Yes & Yes \\
\hline 13 & 6 & 2011 & Hemorrhage & Rt frontal & I & Yes & Unable \\
\hline 14 & 10 & 2012 & Hemorrhage/GCS & Rt frontal & III & Yes & No \\
\hline 15 & 10 & 2012 & Follow-up & Lt frontal & I & Yes & No \\
\hline 16 & 2 & 2012 & Hemorrhage/GCS & Rt frontal & IV & Yes & No \\
\hline 17 & 15 & 2012 & Incidental finding & Rt frontal & III & Yes & Yes \\
\hline 18 & 7 & 2013 & Hemorrhage & Rt insular & III & Yes & Yes \\
\hline 19 & 10 & 2013 & Incidental finding & Rt sylvian & II & Yes & Yes \\
\hline 20 & 16 & 2013 & Hemorrhage & Rt parietal & I & Yes & Unable \\
\hline
\end{tabular}

TABLE 2. Summary of functional status in 19 patients with AVMs surgically treated*

\begin{tabular}{|c|c|c|c|c|c|}
\hline \multirow[b]{2}{*}{ Case No. } & \multirow[b]{2}{*}{ SM Grade } & \multicolumn{3}{|c|}{ Functional Status (MRC grade, muscle power) } & \multirow{2}{*}{$\begin{array}{c}\mathrm{mRS} \text { Score at } \\
6 \mathrm{Mos}\end{array}$} \\
\hline & & Preop & Immediately Postop & 6 Mos Postop & \\
\hline 1 & IV & Lt hemiparesis $(4 / 5)$ & Unchanged & Unchanged & 1 \\
\hline 2 & II & Rt hemiparesis $(4 / 5)$ \& dysphasia & Unchanged & Rt hemiparesis (4/5) w/ resolved dysphasia & 1 \\
\hline 3 & III & Lt hemiparesis $(3 / 5)$ & Unchanged & Lt hemiparesis $(4 / 5)$ & 1 \\
\hline 4 & II & Normal & Normal & Normal & 0 \\
\hline 5 & II & Seizures only & Unchanged & Normal w/o anticonvulsants & 0 \\
\hline 6 & III & Lt hemiparesis $(1 / 5)$ & Lt hemiparesis $(3 / 5)$ & Lt hemiparesis $(4 / 5)$ & 1 \\
\hline 7 & II & Normal & Normal & Normal & 0 \\
\hline 8 & II & Normal & Normal & Normal & 0 \\
\hline 9 & III & Normal & Normal & Normal & 0 \\
\hline 10 & III & Seizures only & Normal & Seizures controlled w/ anticonvulsants & 1 \\
\hline 11 & IV & Quadrantanopia only & Unchanged & Normal & 0 \\
\hline 12 & IV & Lt hemiparesis $(3 / 5)$ & Unchanged & Lt hemiparesis $(4 / 5)$ & 1 \\
\hline 13 & 1 & Normal & Normal & Normal & 0 \\
\hline 14 & III & Lt hemiparesis $(4 / 5)$ & Unchanged & Unchanged & 1 \\
\hline 15 & I & Normal & Normal & Normal & 0 \\
\hline 16 & IV & Normal & Normal & Normal & 0 \\
\hline 17 & III & Normal & Lt arm weakness $(3 / 5)$ & Normal & 0 \\
\hline 18 & III & Lt hemiparesis $(0 / 5)$ & Unchanged & Lt hemiparesis $(3 / 5)$ & 2 \\
\hline 19 & II & Normal & Lt hemiparesis $(3 / 5)$ & Normal & 0 \\
\hline 20 & I & Normal & Normal & Normal & 0 \\
\hline
\end{tabular}

MRC $=$ Medical Research Council.

* At 6 months after surgery, complete resection of the AVM nidus was achieved in all cases. 
ing a score of 0 (12 cases) or 1 ( 7 cases). No new long-term neurological deficits occurred following surgery.

The combination of neuronavigation and intraoperative Doppler ultrasonography at our institution allowed the AVM nidus to be completely resected in all cases, as demonstrated on angiography at 6 months postoperatively. Arteriovenous malformation resections in Cases 8 and 15 were performed in the same patient but in two separate locations and 3 years apart. This interesting case arose because the angiography study 6 months after the first resection demonstrated a possible new AVM arising in a different location; however, the AVM resection was complete. The new AVM in this patient was followed with angiography over the next 2 years, and because it continued to slowly enlarge, further surgery was undertaken in 2012 (Table 1).

\section{Illustrative Cases}

The following 2 cases demonstrate some of the benefits of combining neuronavigation with intraoperative ultrasonography during microscopic AVM dissection in our series.

\section{Case 14}

\section{History and Examination}

This 10-year-old girl presented in 2012 with a reduced GCS score attributable to a large right frontal intracerebral hematoma (Fig. 1). Urgent surgery was undertaken given the patient's rapidly declining level of consciousness, and because of extensive intraoperative blood loss, a clip was placed on a middle cerebral artery (MCA) AVM feeder to gain hemorrhage control. Decompressive craniectomy and prolonged intracranial pressure monitoring with sedation were performed ${ }^{15}$ following intracerebral clot evacuation, but AVM resection was not attempted by the attending neurosurgeon. After a prolonged intensive care unit stay, she made a good recovery and had only mild contralateral hemiparesis (Table 2) prior to definitive AVM surgery 12 weeks later. Preoperative angiography (Fig. 2) demonstrated an SM Grade III AVM arising from the anterior cerebral artery and MCA and draining to the vein of Trolard, and the previously placed clip could also be seen. This AVM was believed to be unsuitable for endovascular embolization by our neuroradiology department.

\section{Operation}

Following a family discussion regarding treatment options, we attempted microsurgical removal of the AVM using a custom-made titanium cranioplasty insertion to cover the previous decompressive craniectomy site. Initial Doppler ultrasound and anatomical views from the extradural space are shown in Fig. 3.

The challenges in this particular case were the volume of surrounding encephalomalacia due to the initial hematoma and the resulting large CSF spaces, which could have rendered neuronavigation very inaccurate once the dura mater was opened. As shown in Fig. 4, the microscopic dissection was guided by the neuronavigation heads-up display object, and any inaccuracy could have resulted in incomplete resection or resection through an adjacent elo-

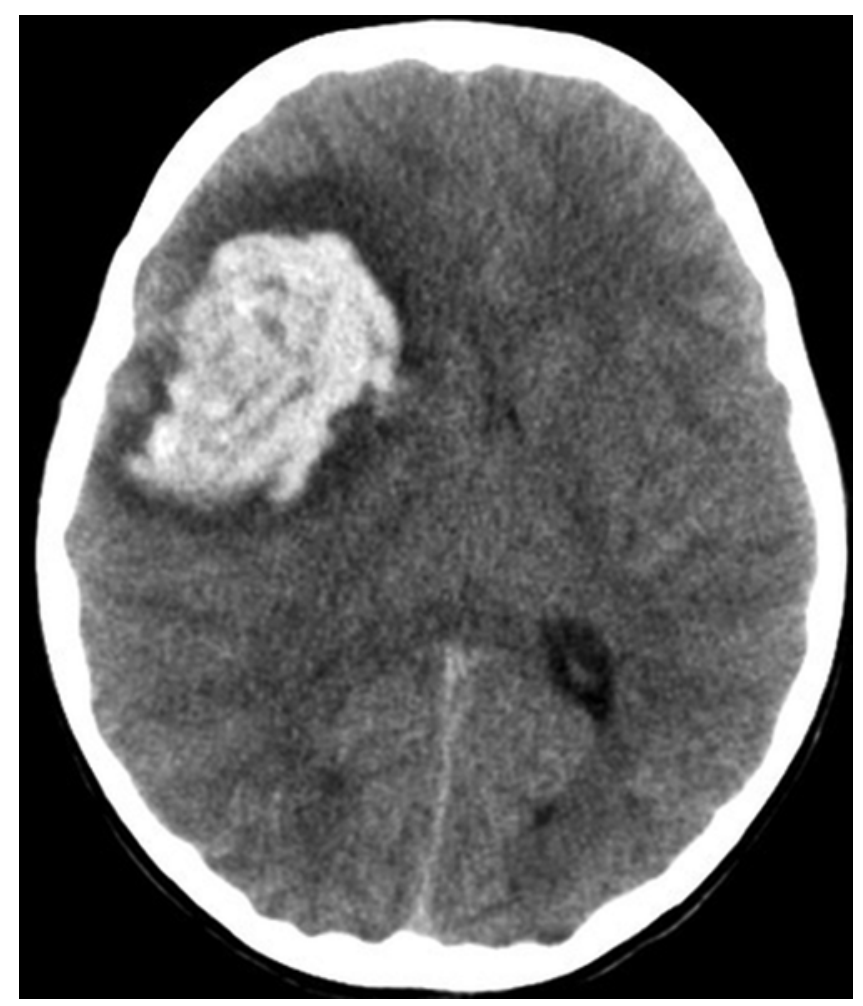

FIG. 1. Case 14. Presentation brain CT showing a large insular region hematoma with significant associated mass effect.

quent area. Indeed, Fig. 5, shows that upon opening the dura and allowing CSF drainage, the previously marked nidus (object outlined in red in Fig. 3) is no longer accurate, even though to the operating surgeon the microscope (blue crosshair) is focused on the AVM.

To adjust for this intraoperative shift, we used the integrated Doppler ultrasound to accurately move the marked object (red-outlined object indicating the AVM nidus) over the actual AVM (Fig. 5). This allowed the operator to clearly see the object on the projected microscope overlay and therefore helped to prevent inaccurate dissection. As can be seen from Fig. 6, the projected object position was found to be inaccurate by nearly $2 \mathrm{~cm}$.

Complete resection of the AVM nidus was achieved using an integrated microscope heads-up display (Fig. 4). The feeding arteries could be demonstrated in great detail on Doppler ultrasound throughout the procedure (Figs. 7 and 8). The ultrasound also allowed assessment of any hemorrhage within the ventricular system that may obstruct the foramen of Monro when operating close to the ventricles, as in this case. Complete AVM resection was confirmed using Doppler ultrasound (Fig. 9). A custommade titanium cranioplasty was placed at the end of the procedure to cover the previous bony decompression.

\section{Postoperative Course}

Complete AVM resection was confirmed on angiography at 6 months after treatment. There was no change in the patient's neurological function immediately postoperatively or at the 6-month review (Table 2). 

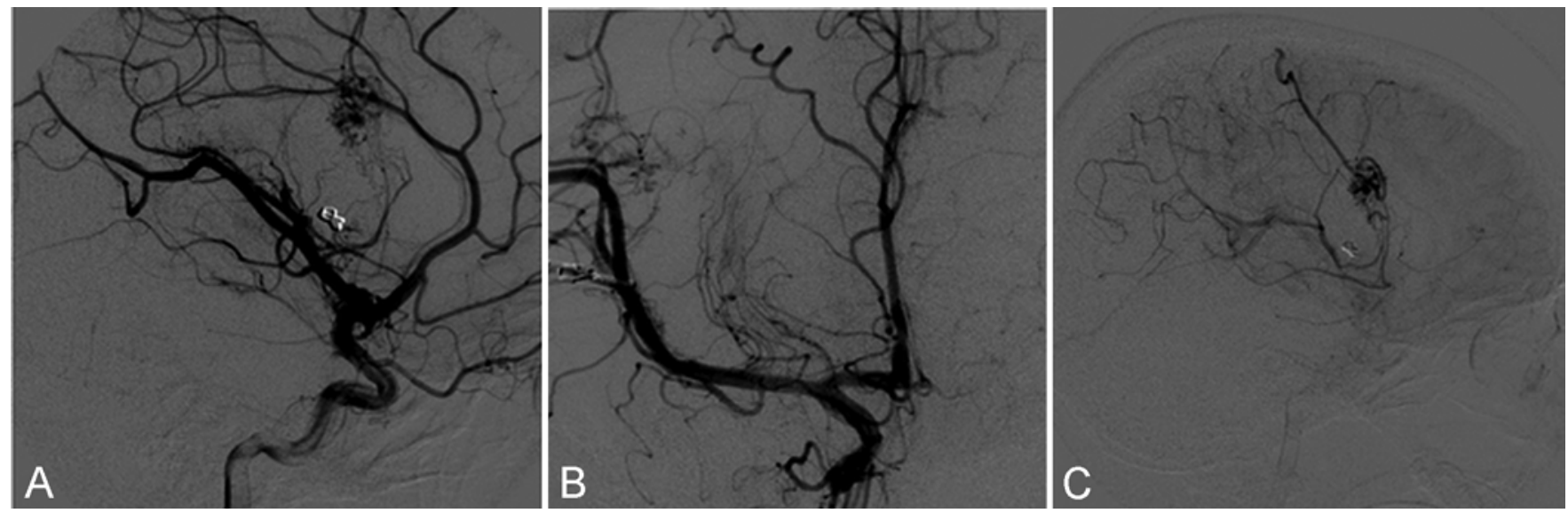

FIG. 2. Case 14. Right internal carotid artery angiograms, lateral (A) and anteroposterior (B) views, showing the AVM arising from the anterior cerebral and lenticulostriate arteries. Venous drainage was to the vein of Trolard, as shown on the venous phase angiogram (C). The surgical clip placed on admission can also be identified on an MCA vessel.

\section{Case 18}

History and Examination

This 7-year-old boy presented in 2013 with left hemiparesis following a right insular region and intraventricular hemorrhage. Initial imaging was performed (Fig. 10), and immediate management consisted of the placement of an external ventricular drain. Angiography demonstrated an SM Grade III AVM supplied by the right MCA and lenticulostriate artery with venous drainage to the vein of Labbé (Fig. 11). An intranidal aneurysm was embolized with coils, and the AVM was partially embolized (Fig. 12).

\section{Operation}

Following successful angiography and a family discussion regarding treatment options, the patient proceeded to surgery. As in the last case, our standard operative setup was neuronavigation (Brainlab AG) linked to Doppler ultrasound (Hitachi Aloka ProSound Alpha 7) utilizing an integrated microscope display (OPMI Pentero, Carl Zeiss). This enabled the operator to "see" the previously marked AVM nidus throughout the dissection (Fig. 4). This minimized surrounding tissue damage and helped to guide the extent of resection, which is especially useful with small

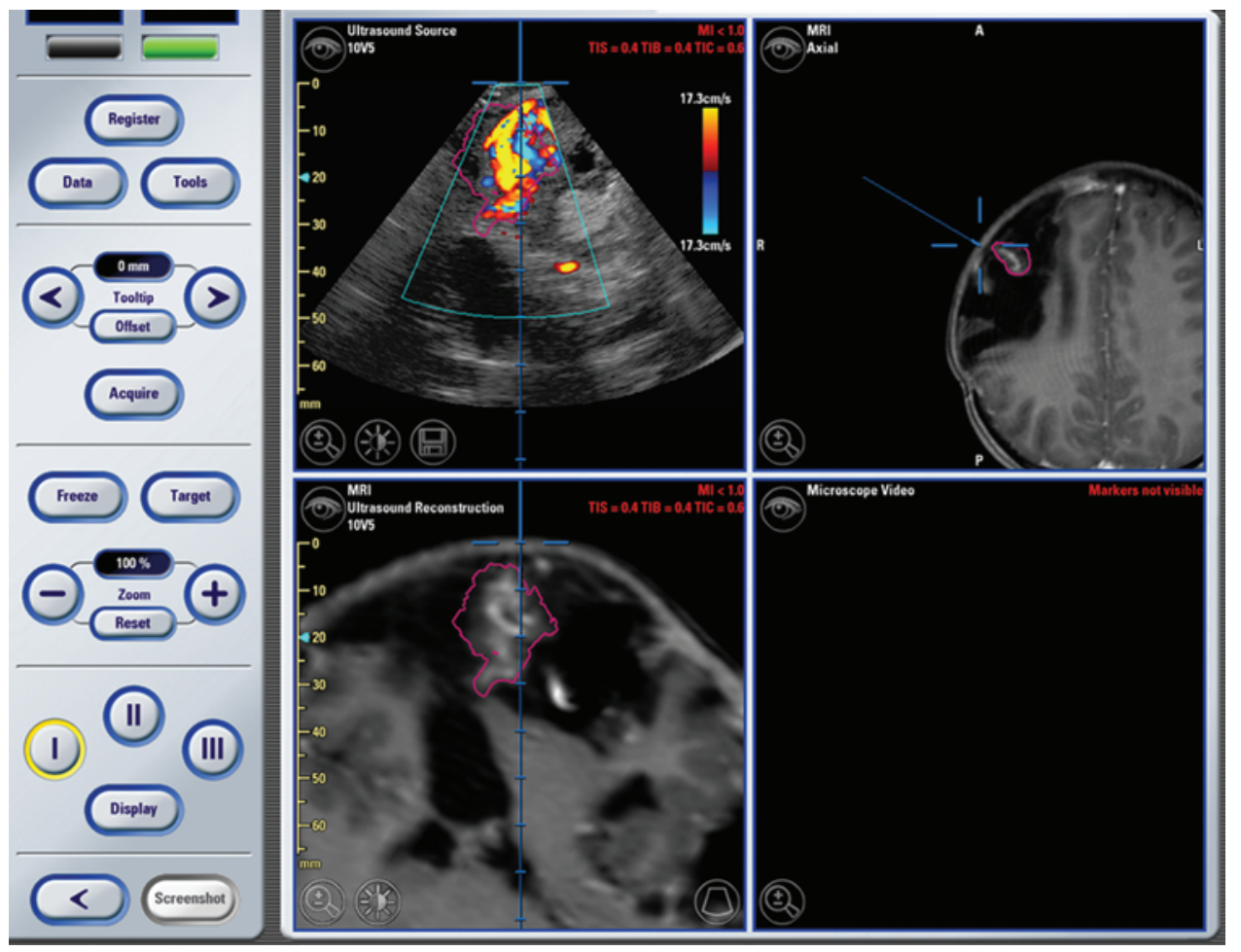

FIG. 3. Case 14. Neuronavigation system screenshot demonstrating the integrated Doppler ultrasonography view of the AVM from the extradural space. The AVM nidus (red-outlined object) was marked on the image guidance software preoperatively, and the microscope was registered as a "pointer" (blue crosshair) to allow accurate dissection. Doppler ultrasound view (upper left), preoperative axial MR image (upper right), and inline trajectory MR view (lower left). Figure is available in color online only. 


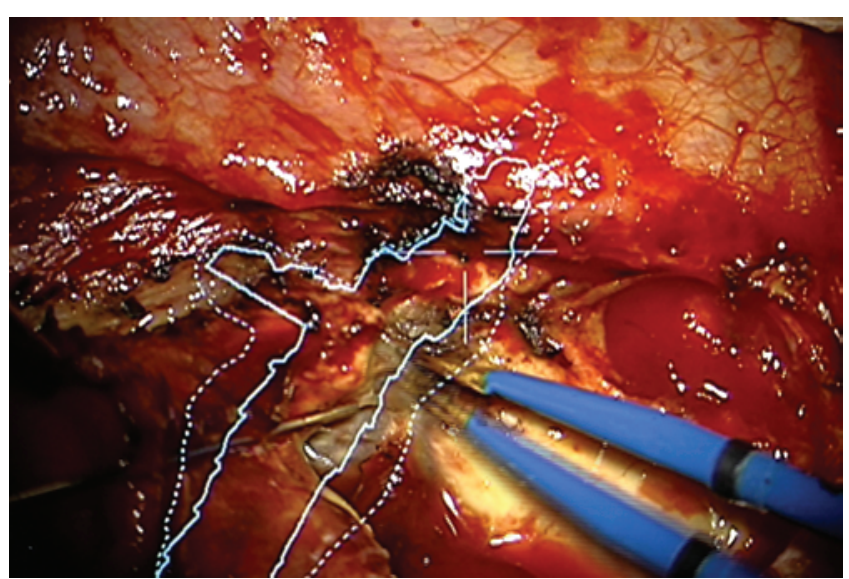

FIG. 4. Case 14. Operating surgeon's microscope view of the AVM with object integration following correction of the object "shift." This allows very precise dissection along the AVM margin, as shown. Solid line indicates the object outline at that depth; dotted line indicates the object outline at deeper depths. Figure is available in color online only.

AVMs. Initial Doppler ultrasonography from the extradural space is shown in Fig. 13. The preoperatively marked nidus can be seen in red on the ultrasound and imaging displays in Fig. 14.

As the dissection progressed the operator was able to visualize the target nidus at all times. The microscope was also integrated into the neuronavigation software as a "pointer" (blue crosshair in Fig. 14) allowing very accurate dissection of the AVM nidus, which is critical in small AVMs as in this case. The AVM was resected using stan-

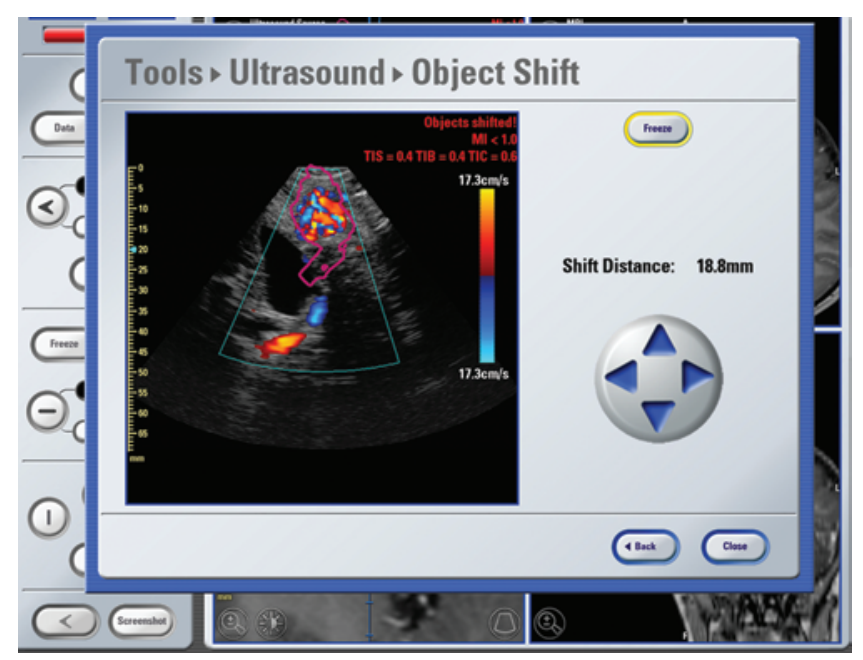

FIG. 6. Case 14. Neuronavigation system screenshot demonstrating the use of the Doppler ultrasound to correct for object shift due to CSF loss. Figure is available in color online only.

dard microsurgical techniques, and Doppler ultrasound was used to confirm the extent of resection.

As can be seen in Fig. 15, a very small separate nidus was seen on Doppler ultrasonography approximately 1 $\mathrm{cm}$ superior and deep to the previous dissection. This appeared to be in the location of the lenticulostriate branch (white arrow in Fig. 11 right), but there was now clearly a nidus, which had not been seen on the angiography study obtained several weeks before surgery. This nidus was marked as a separate target (Fig. 14). The microscope tra-

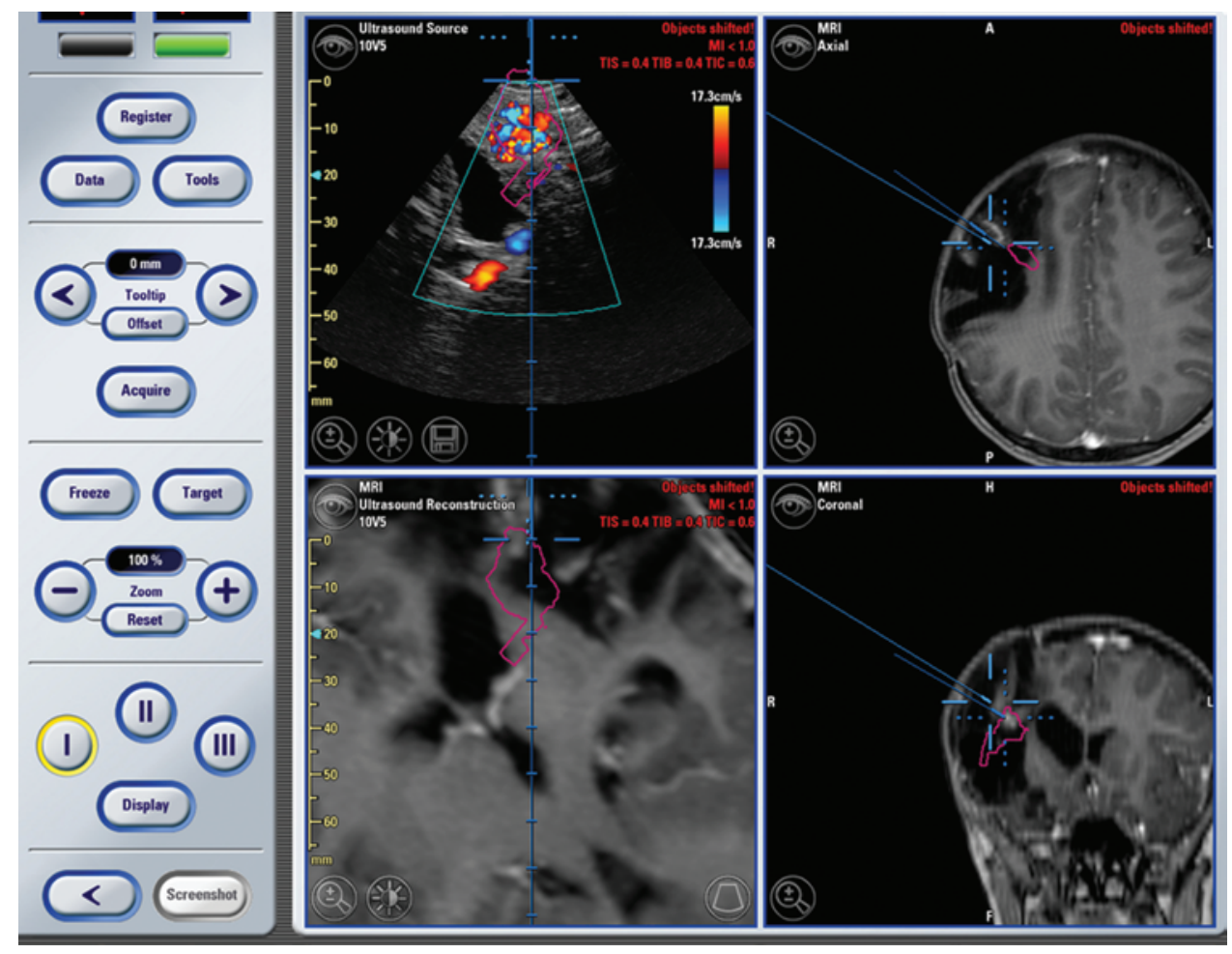

FIG. 5. Case 14. Neuronavigation system screenshot demonstrating shift of the AVM nidus (red-outlined object) once the dura was opened. Ultrasound image (upper left), axial MR image (upper right), coronal MR image (lower right), and inline trajectory MR view (lower left). Figure is available in color online only. 


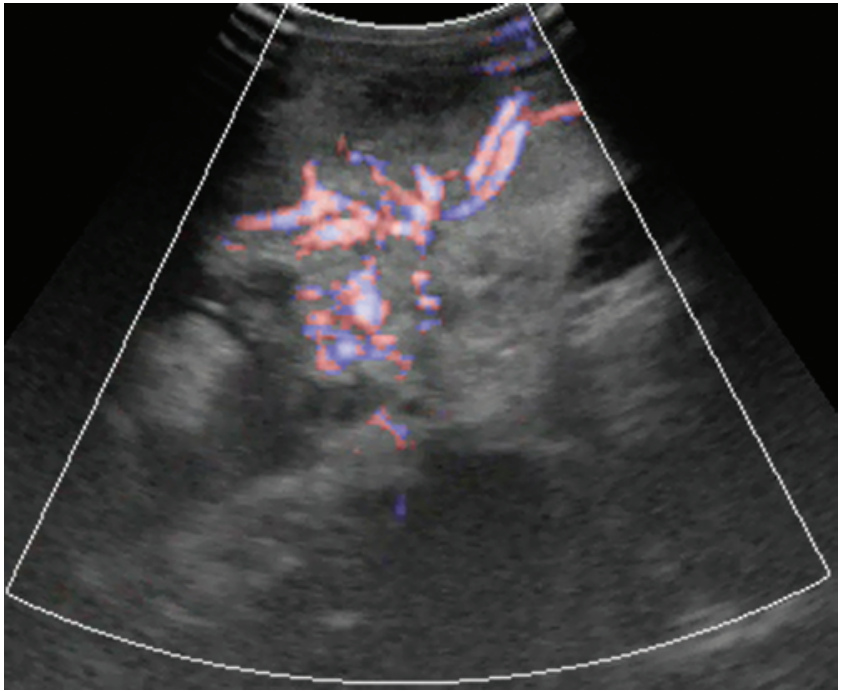

FIG. 7. Case 14. Doppler ultrasound view of the feeding lenticulostriate arteries and the AVM nidus prior to resection from the extradural space. Figure is available in color online only.

jectory (green line in Fig. 14) was then adjusted to allow direct dissection of this lesion.

The newly identified nidus was found intraoperatively using this technique (Fig. 14), enabling complete resection of this separate deep AVM component. Following microscopic resection of this deep component, Doppler ultrasound was again used to confirm extent of resection (Fig. 16).

\section{Postoperative Course}

Without the use of Doppler ultrasound in this case, the additional AVM component would have remained and exposed the patient to further intervention in the future. We believe that the integrated use of the above technology allowed us to identify and resect these 2 small AVMs safely with the most minimal brain retraction and dissection pos-

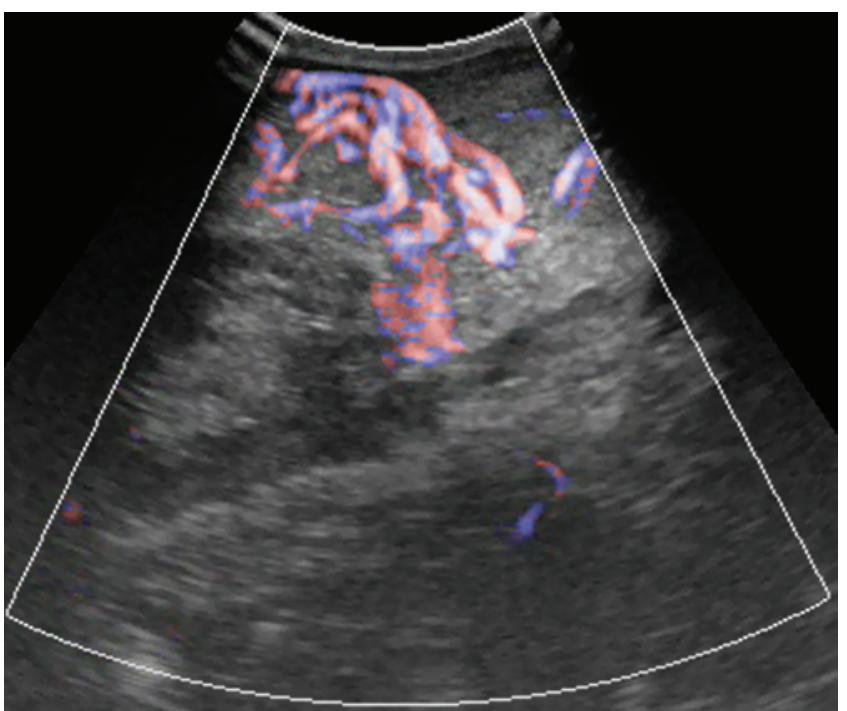

FIG. 8. Case 14. Doppler ultrasound view of the superficial AVM nidus as visualized from the brain surface. Figure is available in color online only.

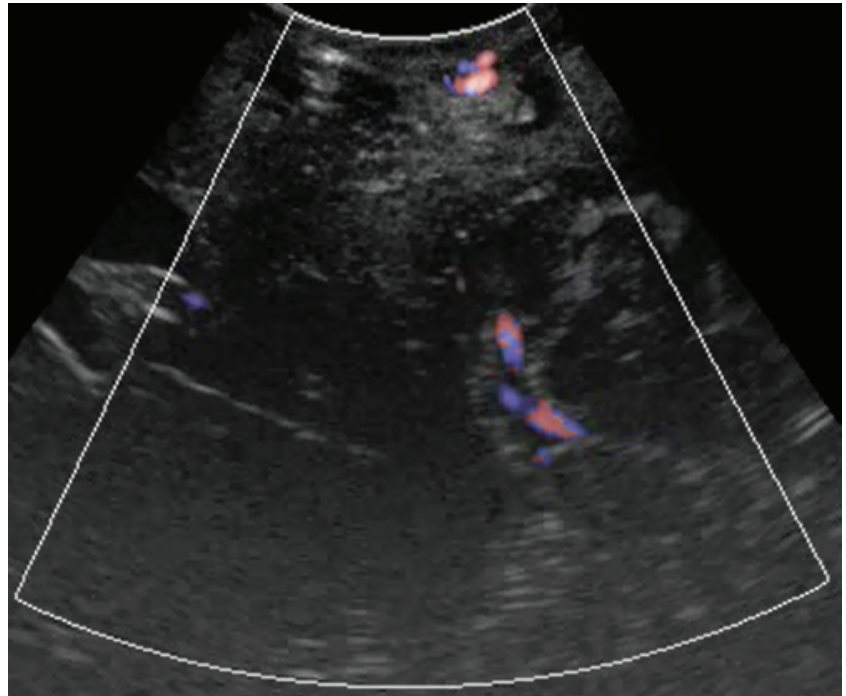

FIG. 9. Case 14. Doppler ultrasound view from the brain surface showing complete AVM nidus resection and no hematoma within the ventricular system. Figure is available in color online only.

sible. There was no change in neurological function immediately postoperatively, but useful improvement was seen at the 6-month follow-up (Table 2). Complete AVM resection was also confirmed by angiography at the 6-month follow-up.

\section{Discussion}

The aim of treating a pediatric AVM should be complete obliteration considering the high risk of hemorrhage

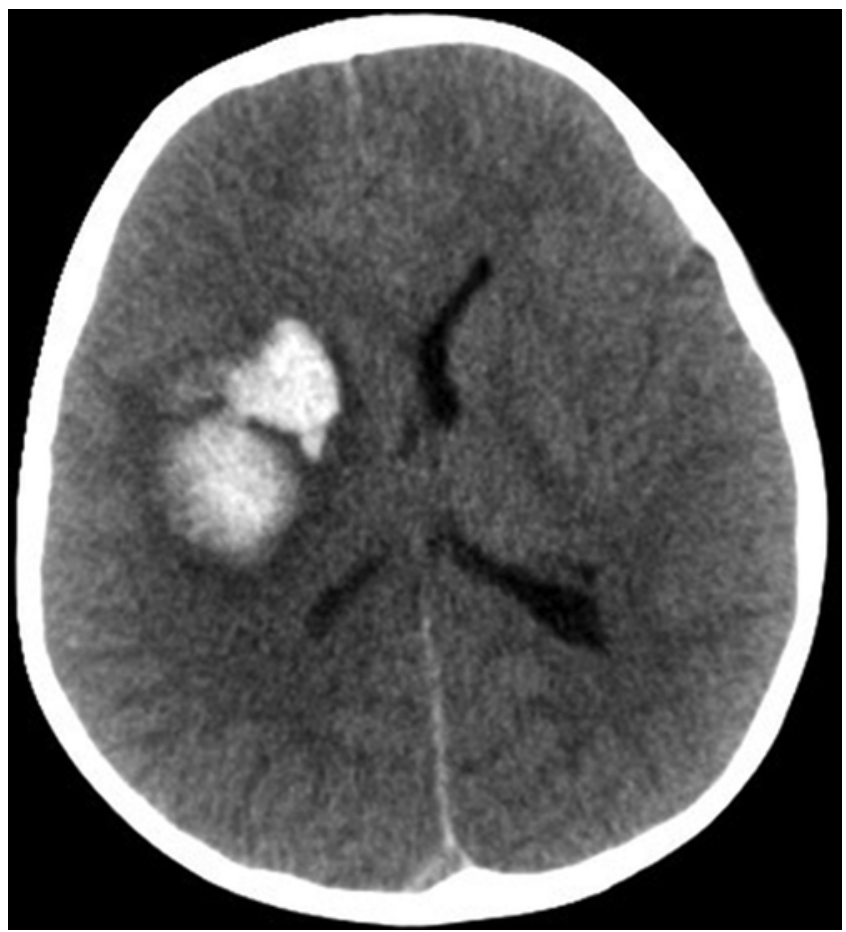

FIG. 10. Case 18. Presentation brain CT demonstrating basal ganglia hemorrhage. There was also an associated intraventricular hemorrhage not visible on this axial slice. 

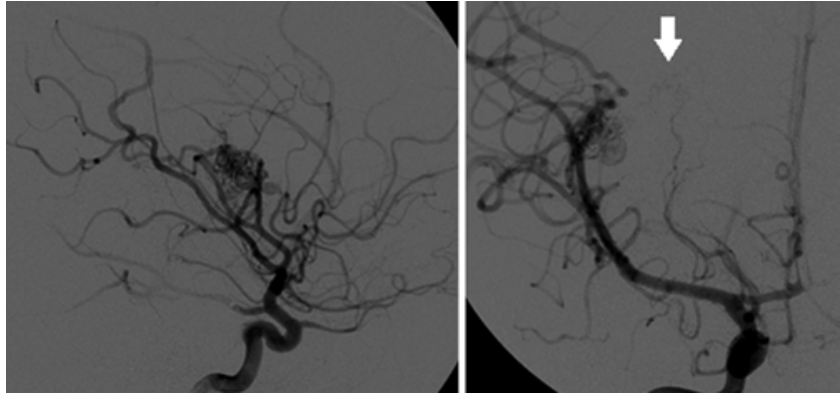

FIG. 11. Case 18. Right carotid artery angiograms, lateral (left) and anteroposterior (right) views, showing the AVM nidus being fed from the right MCA and lenticulostriate artery. There was a tiny feeding branch of the lenticulostriate artery (white arrow).

as well as the morbidity and mortality associated with this lesion. ${ }^{16}$ Recent technological advances now allow multimodality treatments of an AVM including microsurgical, endovascular, and stereotactic interventions. Overall, mortality from pediatric AVM has reduced to around $4.5 \%-$ $12 \%$ depending on lesion location. ${ }^{11,13}$ Operative intervention for pediatric AVM remains an extremely challenging undertaking because of the lower blood volumes and the higher general anesthesia and future hemorrhage risks encountered in this population.

Results of our series show presentation rates similar to those in other published series, with around $80 \%$ of children presenting with hemorrhage. ${ }^{11,13,16}$ No deaths were recorded in our series, nor were any long-term new neurological deficits, although 2 patients had transient contralateral weakness immediately postoperatively. This rate of good functional outcome is similar to that noted by Nair et al., ${ }^{16}$ who found that $86 \%$ of patients had a favorable outcome (mRS Score $\leq 2$ ).

Many authors have described the intraoperative use of ultrasound with and without Doppler to help guide resection in the adult population, , ,4,6,8,10,12,20,22-24,26,27,28 while others have shown the benefits of integrated neuronavigation. ${ }^{1,14,22}$ Intraoperative angiography and indocyanine green video-angiography have also been used in conjunction with intraoperative Doppler ultrasound to aid AVM

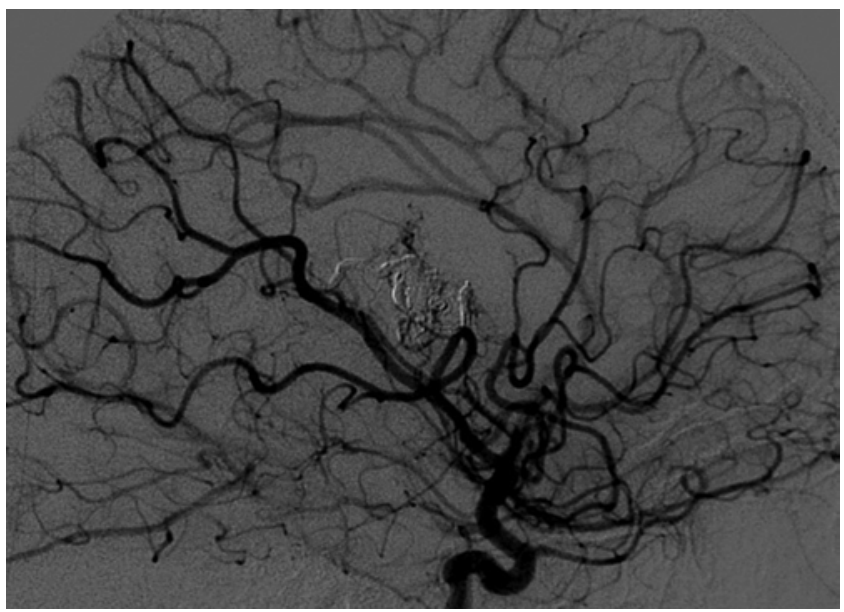

FIG. 12. Case 18. Right carotid artery angiogram showing coiling of an intranidal aneurysm and partial embolization of the AVM nidus.

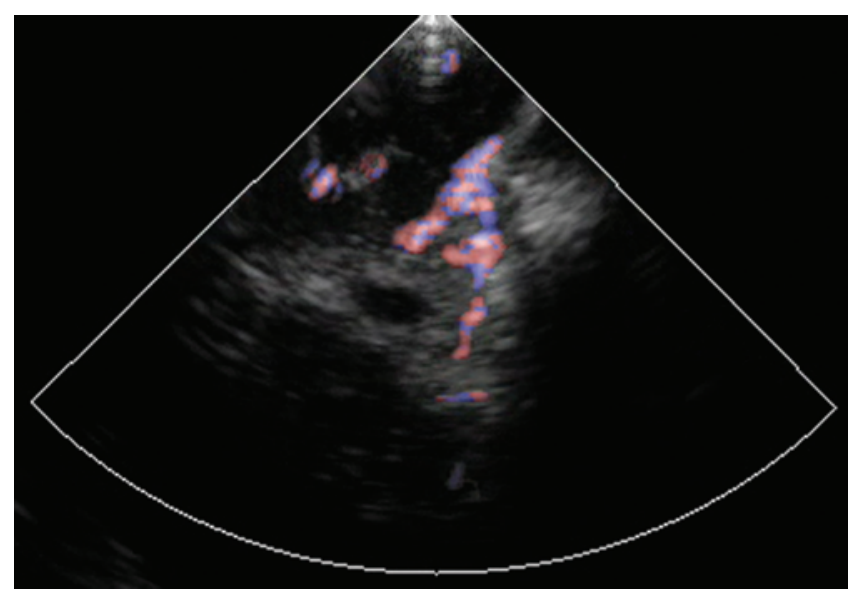

FIG. 13. Case 18. Doppler ultrasound view from the brain surface clearly showing the feeding artery with the AVM nidus. Figure is available in color online only.

resection in the adult population. ${ }^{2,19,27}$ Several authors have reported the use of multimodality treatment in the pediatric population, $3,5,9,11,13,16,17,19,25$ including neuronavigation, ultrasound, or intraoperative angiography. Complete resection rates are all high in the published series, ranging from $80 \%$ to $100 \%$. $5,9,13,16,21$

We believe the use of intraoperative ultrasound in combination with neuronavigation and integrated operative microscopy is a safe and reliable technique to aid AVM resection in pediatric patients. There are clear advantages in being able to position a smaller craniotomy directly over the intended target lesion: it minimizes blood loss from the craniotomy site and reduces incision length. Doppler ultrasound also allows early identification of the arterial feeders and gives the surgeon an overall impression of the flow dynamics prior to dural opening. The surgeon's ability to have neuronavigation overlay during the dissection greatly helps to minimize brain retraction and damage to surrounding tissue. As shown in Case 14, ultrasound can also be used to help orient the dissection should the neuronavigation system lose accuracy because of swelling or CSF loss. A final use of Doppler ultrasound is to check for residual or previously unidentified nidus, as shown in Case 18. The careful use of integrated neuronavigation and Doppler ultrasound during surgery to search for any residual nidus was successful in ensuring complete resection in all 20 cases. Other advantages of ultrasound are the ability to identify any hematoma or hydrocephalus development during surgery and to guide external ventricular drain placement in the presence of small ventricles or intraventricular hemorrhage. This technology is relatively inexpensive, and no morbidity was attributed to its use in our series. While intraoperative and 24-hour postresection angiography represent the gold-standard surgical adjunct, the combination is expensive, invasive, and unavailable at many institutions, such as ours. A recent paper from Gaballah et al. ${ }^{9}$ demonstrated a $5 \%$ complication rate and $14 \%$ AVM recurrence rate using intraoperative angiography in the pediatric population. There is, however, a learning curve in using intraoperative Doppler ultrasonography as well as the associated neuronavigation and microscope software to their full potential, and the initial cost of the technology may be prohibitive at some institutions. Despite the intraoperative accuracy of Doppler ultrasound, 


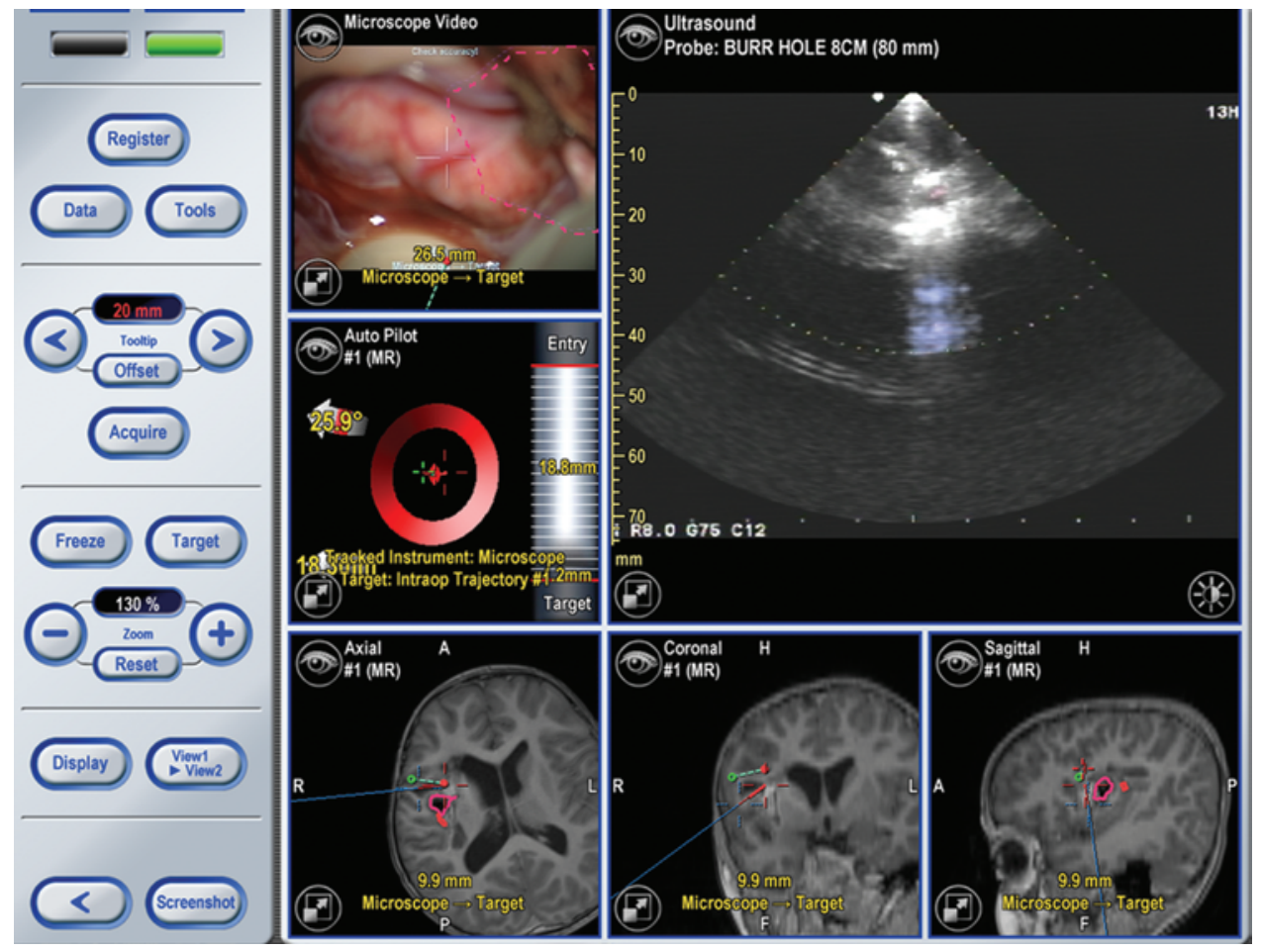

FIG. 14. Case 18. Neuronavigation system screenshot demonstrating acquisition of a new "target" AVM nidus (red crosshair). To aid dissection, a trajectory (green line) was created based on the previous Doppler ultrasound findings. The focus depth of the microscope is registered as a pointer (blue crosshair) to accurately identify the nidus, as shown in the microscopic view (upper left) and axial, coronal, and sagittal MR images (lower), respectively. The originally identified and resected nidus is indicated by red outline in the microscopic and MRI views. Figure is available in color online only.

we believe that 6 months' delayed angiography is mandatory to exclude any residual AVM and to assess for any de novo AVM formation, which is described in the pediatric population ${ }^{7,11,18}$ and indeed in our series (Cases 8 and 15).

\section{Conclusions}

Intraoperative Doppler ultrasonography in combination with neuronavigation and integrated operative microscopy

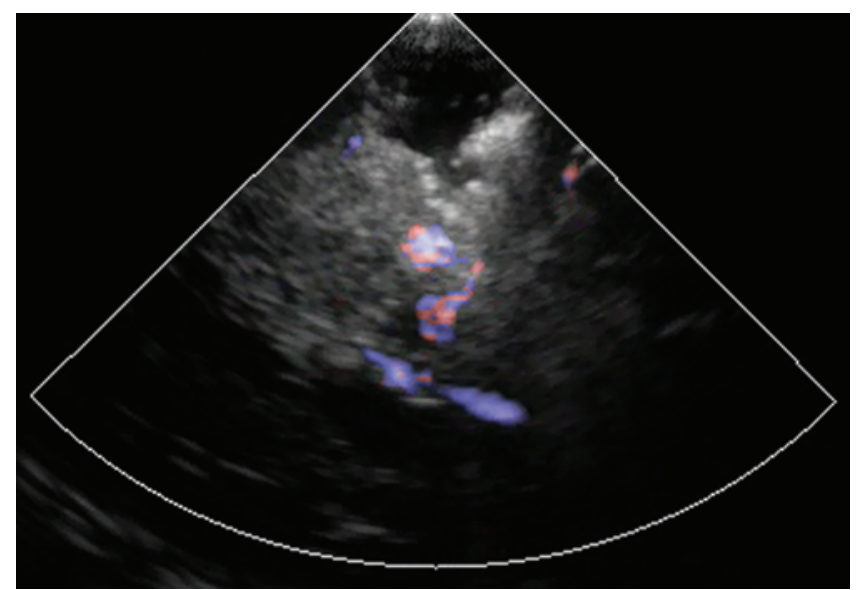

FIG. 15. Case 18. Doppler ultrasound image following complete resection of the AVM nidus initially approached. A separate deeper nidus not seen on preoperative angiography is now visible deep to the initial resection bed shown superiorly. Figure is available in color online only. is a reliable and useful method for intraoperative localization and guidance in AVM resection in the pediatric population. This technique has shown a very high complete resection rate with extremely low associated morbidity and mortality. This technology also has several other applications in aiding the acute surgical management of AVMs in the pediatric population and represents an alternative to on-table angiography, which is not available at many institutions.

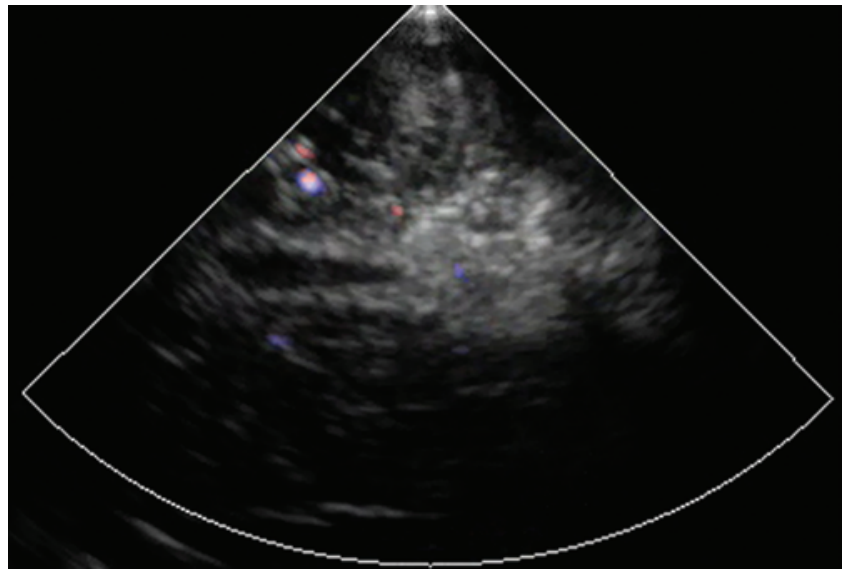

FIG. 16. Case 18. Doppler ultrasound image from the brain surface demonstrating complete resection of the newly identified deeper AVM nidus. Figure is available in color online only. 


\section{References}

1. Akdemir H, Oktem S, Menkü A, Tucer B, Tuğcu B, Günaldi $\mathrm{O}$ : Image-guided microneurosurgical management of small arteriovenous malformation: role of neuronavigation and intraoperative Doppler sonography. Minim Invasive Neurosurg 50:163-169, 2007

2. Black KL, Rubin JM, Chandler WF, McGillicuddy JE: Intraoperative color-flow Doppler imaging of AVM's and aneurysms. J Neurosurg 68:635-639, 1988

3. Caldarelli M, Di Rocco C, Iannelli A, Rollo M, Tamburrini G, Velardi F: Combined management of intracranial vascular malformations in children. J Neurosurg Sci 41:315-324, 1997

4. Cokluk C, Iyigün O, Senel A, Celik F, Rakunt C: The guidance of intraoperative ultrasonography in the surgical treatment of arteriovenous malformation. Minim Invasive Neurosurg 46:169-172, 2003

5. Darsaut TE, Guzman R, Marcellus ML, Edwards MS, Tian L, Do HM, et al: Management of pediatric intracranial arteriovenous malformations: experience with multimodality therapy. Neurosurgery 69:540-556, 2011

6. Dempsey RJ, Moftakhar R, Pozniak M: Intraoperative Doppler to measure cerebrovascular resistance as a guide to complete resection of arteriovenous malformations. Neurosurgery 55:155-161, 2004

7. Frisoli FA, Lang SS, Vossough A, Cahill AM, Heuer GG, Dahmoush HM, et al: Intrarater and interrater reliability of the pediatric arteriovenous malformation compactness score in children. J Neurosurg Pediatr 11:547-551, 2013

8. Fu B, Zhao JZ, Yu LB: The application of ultrasound in the management of cerebral arteriovenous malformation. Neurosci Bull 24:387-394, 2008

9. Gaballah M, Storm PB, Rabinowitz D, Ichord RN, Hurst RW, Krishnamurthy G, et al: Intraoperative cerebral angiography in arteriovenous malformation resection in children: a single institutional experience. J Neurosurg Pediatr 13:222-228, 2014

10. Griffith S, Pozniak MA, Mitchell CC, Ledwidge ME, Dempsey R, Peters A, et al: Intraoperative sonography of intracranial arteriovenous malformations: how we do it. J Ultrasound Med 23:1065-1072, 2004

11. Humphreys RP, Hoffman HJ, Drake JM, Rutka JT: Choices in the 1990s for the management of pediatric cerebral arteriovenous malformations. Pediatr Neurosurg 25:277-285, 1996

12. Kane RA: Intraoperative ultrasonography: history, current state of the art, and future directions. J Ultrasound Med 23:1407-1420, 2004

13. Kondziolka D, Humphreys RP, Hoffman HJ, Hendrick EB, Drake JM: Arteriovenous malformations of the brain in children: a forty year experience. Can J Neurol Sci 19:40-45, 1992

14. Mathiesen T, Peredo I, Edner G, Kihlström L, Svensson M, Ulfarsson E, et al: Neuronavigation for arteriovenous malformation surgery by intraoperative three-dimensional ultrasound angiography. Neurosurgery 60 (4 Suppl 2):345-351, 2007

15. Meyer PG, Orliaguet GA, Zerah M, Charron B, Jarreau MM, Brunelle F, et al: Emergency management of deeply comatose children with acute rupture of cerebral arteriovenous malformations. Can J Anaesth 47:758-766, 2000

16. Nair AP, Kumar R, Mehrotra A, Srivastava AK, Sahu RN, Nair P: Clinical, radiological profile and outcome in pediatric Spetzler-Martin grades I-III arteriovenous malformations. Childs Nerv Syst 28:593-598, 2012

17. Niazi TN, Klimo P Jr, Anderson RC, Raffel C: Diagnosis and management of arteriovenous malformations in children. Neurosurg Clin N Am 21:443-456, 2010
18. Ogilvy CS, Stieg PE, Awad I, Brown RD Jr, Kondziolka D, Rosenwasser R, et al: AHA Scientific Statement: Recommendations for the management of intracranial arteriovenous malformations: a statement for healthcare professionals from a special writing group of the Stroke Council, American Stroke Association. Stroke 32:1458-1471, 2001

19. Pietilä TA, Stendel R, Jansons J, Schilling A, Koch HC, Brock M: The value of intraoperative angiography for surgical treatment of cerebral arteriovenous malformations in eloquent brain areas. Acta Neurochir (Wien) 140:1161-1165, 1998

20. Rubin JM, Hatfield MK, Chandler WF, Black KL, DiPietro MA: Intracerebral arteriovenous malformations: intraoperative color Doppler flow imaging. Radiology 170:219-222, 1989

21. Sanchez-Mejia RO, Chennupati SK, Gupta N, Fullerton H, Young WL, Lawton MT: Superior outcomes in children compared with adults after microsurgical resection of brain arteriovenous malformations. J Neurosurg 105 (2 Suppl):82-87, 2006

22. Unsgaard G, Ommedal S, Rygh OM, Lindseth F: Operation of arteriovenous malformations assisted by stereoscopic navigation-controlled display of preoperative magnetic resonance angiography and intraoperative ultrasound angiography. Neurosurgery 56 (2 Suppl):281-290, 2005

23. Wang S, Liu L, Zhao YL, Zhang D, Wang R, Zhao JZ: [Strategy for assisted cerebral arteriovenous malformation surgery.] Zhonghua Yi Xue Za Zhi 90:869-873, 2010 (Chinese)

24. Wang Y, Wang Y, Wang Y, Taniguchi N, Chen XC: Intraoperative real-time contrast-enhanced ultrasound angiography: a new adjunct in the surgical treatment of arteriovenous malformations. J Neurosurg 107:959-964, 2007

25. Westra SJ, Curran JG, Duckwiler GR, Zaninovic A, Hall TR, Martin NA, et al: Pediatric intracranial vascular malformations: evaluation of treatment results with color Doppler US. Work in progress. Radiology 186:775-783, 1993

26. Woydt M, Perez J, Meixensberger J, Krone A, Soerensen N, Roosen K: Intra-operative colour-duplex-sonography in the surgical management of cerebral AV-malformations. Acta Neurochir (Wien) 140:689-698, 1998

27. Zhang Y, Wang S, He W: [Intraoperative Doppler ultrasonography and ultrasound angiography in operation for brain arteriovenous malformations.] Zhonghua Yi Xue Za Zhi 88:2461-2464, 2008 (Chinese)

28. Zhou DB, Zhao JZ, Wang S, Ni M, Wang R, Zhang D, et al: [Application of intraoperative real-time ultrasound in the surgical treatment of cerebral arteriovenous malformation.] Zhonghua Yi Xue Za Zhi 85:1688-1691, 2005 (Chinese)

\section{Author Contributions}

Conception and design: Walkden, Kamaly-Asl. Acquisition of data: Walkden, Herwadkar, Kamaly-Asl. Analysis and interpretation of data: Walkden, Herwadkar. Drafting the article: Walkden. Critically revising the article: Zador, Herwadkar, Kamaly-Asl. Reviewed submitted version of manuscript: Zador, Herwadkar. Administrative/technical/material support: Zador, Herwadkar. Study supervision: Kamaly-Asl.

\section{Correspondence}

James Walkden, Departments of Neurosurgery and Neuroradiology, Royal Manchester Children's Hospital, Oxford Rd., Manchester M13 9WL, United Kingdom. email: jwalkden@ doctors.net.uk. 